\title{
A Survey of Energy Efficient Mobile Sensor Dispatch in Wireless Sensor Network
}

\author{
M. Balaganesh \\ Asso.Professor, Dept of CSE \\ Sembodai Rukmani Varatharajan Engineering \\ College, Sembodai, Tamilnadu
}

\author{
R. Maria Johnson \\ PG Scholar, Dept of CSE \\ Sembodai Rukmani Varatharajan Engineering \\ College, Sembodai, Tamilnadu
}

\begin{abstract}
The survey tires to review the sensor dispatch in wireless sensor network. The wireless sensor networks contain static nodes and mobile nodes. In this paper we discuss movement of multi attribute mobile path and the issues of travelling path of mobile sensor. Mobility in wireless sensor networks is controllable and cooperative. The mobile sensor dispatch to the event location without any obstacles to reach the location and travelling in a shortest path. The wireless sensor network scheduled to the travelling path of mobile sensor as to extend a life time using multi round multi attribute sensor. MAM sensors are equipped with multiple sensing devices and can visit event locations to perform more in depth analysis.
\end{abstract}

\section{Keywords}

MSDD, multi-round multi-attribute sensor, MANET, Multirobot system, MAM sensors

\section{INTRODUCTION}

A WSN is usually deployed with static sensors to perform monitoring missions. However, due to the dynamics of events or environments, a purely static WSN could face these challenges: (1) Sensors are often scattered in a ROI by aircrafts or robots. These randomly scattered sensors could not guarantee complete coverage of the Roland may be partitioned into disconnected sub networks. The existence of obstacles could even worsen the problem (2). Sensors are usually powered by batteries. Mobile WSN and mobile Adhoc network (MANET) are in essence. Mobility in a MANET is often arbitrary whereas mobility in a mobile WSN should be 'intentional', in the sense that can control their movement to achieve our missions. Our discussion focus on two types of nodes mobile sensors and data ferries [1]. With the former, one may change the network topology by moving these mobile sensors. With the latter, one may maneuver these data ferries to collect or relay sensing data. Hybrid sensor networks with static and mobile nodes open a new frontier of research in wireless sensor networks (WSNs). Static sensors support environmental sensing and network communication. Mobile sensors are more resource rich in sensing and computing capabilities and can move to particular locations to conduct more complicated missions such as repairing the network or providing in-depth analysis. Introducing mobility to a WSN not only reduces its deployment and maintenance costs but also enhances its capability.

\section{RELATED WORK}

Mobility management has received extensive attention in mobile ad hoc networks (MANETs). They consider that nodes move in an arbitrary manner or follow some mobility models. Unlike MANETs, node mobility in hybrid WSNs is controllable and can even be coordinated. A Multi-robot system (MRS), one topic in the field of robots, uses multiple cooperative robots to accomplish a task in an uncertain environment. Multi-agent reinforcement learning is proposed to train robots to learn mappings from their statuses to their actions. Multi-robot task allocation determines which robot should execute which task to cooperatively achieve the overall goal, where a task is viewed as an independent sub goal that is necessary for achieving the overall goal. However, does not aim at the energy issue of robots [2]. The sensor dispatch problem, but they do not consider energy balance and only optimize energy consumption in one round. Some studies deploy mobile sensors to track moving targets. The purserevader game is where a pursuer needs to intercept an evader by the assistance of static sensors. Target tracking with the assistance of mobile sensors is discussed in with concerns of energy consumption, network connectivity and sensing coverage. They assume that the future trajectory of the moving target may be predicted, where as our work allows events to arbitrarily appear. Several variations of the sensor dispatch problem have been studied in the literature[8]. In, static sensors detecting events will ask mobile sensors to move to their locations to conduct more in-depth analysis.

\subsection{Mobile Management of Mobile Sensor}

Sensor deployment is a basic issue since it decides a WSN's detection ability. A good deployment should satisfy both coverage and connectivity. Coverage requires that each location in the ROI be monitored by sensors, and connectivity requires that the network remain not partitioned. With mobile sensors, the deployment job becomes 'automatic'. Introduce three deployment methods as follows.

\subsection{Force-base Deployment}

The force-based deployment images that virtual forces drive sensors to move sensors by virtual forces. In each sensor is viewed as an electron and is repulsed by other sensors. The force from a higher sensor density area is greater than that from a lower density area, and the force from a nearer sensor is greater than that from a farther sensor.

\subsection{Graph-based Deployment}

The graph-based deployment identifies uncovered holes and moves sensors to cover them. The work adopts a Voronoi diagram to search uncovered holes and moves sensors to cover these holes. Given a set of sensors on a 2D plane, the Voronoi diagram consists of a number of Voronoi polygons such that each polygon contains one sensor and the points in the polygon are closer to the interior sensor than to other exterior sensors. When the sensing range of a sensor cannot completely cover its Voronoi polygon, there could be an uncovered hole in that polygon. 


\subsubsection{Assignment-base Deployment}

The assignment-based deployment computes the locations to be placed with sensors and then dispatches them in an energy efficient way. Assignment-Based Deployment focuses on deployment in ROIs with obstacles. It considers two related problems: sensor placement and sensor dispatch. The former asks how to use the minimum number of sensors in a ROI to guarantee coverage and connectivity. The latter asks how to dispatch mobile sensors to the designated locations computed by the placement result such that their moving energy is minimized. To solve the placement problem, partitions a ROI A into single-row and multi-row regions.[7] A single-row region requires one row of sensors to cover it, and a multi row region requires multiple rows of sensors to cover it. Various approaches have been proposed to self-deploy mobile sensors. The virtual force approach (VFA) models the interactions among sensors as a combination of attractive and repulsive forces. As a result of these antagonist forces, the sensors spread throughout the environment. Drawbacks of VFA include complex required tuning of several parameters and an oscillatory behavior of sensors. Possible improvements to decrease the oscillations include the introduction of dissipative forces or the definition of arbitrary thresholds as stopping conditions. The tuning of such thresholds is laborious and relies on an off-line configuration. The virtual force model is also at the basis of several other proposals of these proposals focus on the one presented in under which a dynamically calculate constraint on the length of sensor movements prevents oscillations.

\subsection{MAM Sensor Dispatch Problem}

A set of attributes and a hybrid WSN consisting of static and MAM sensors. Sensors can use GPS (global positioning system) or some localization schemes to obtain their own locations. Static sensors form a connected network that fully covers the sensing field. They cooperate to identify events of the same attribute that could appear anywhere in the sensing field. Thus, each event reported by static sensor(s) is associated with exact one attribute [1]. No assumption on the distribution of event locations. For computation efficiency, divide time into repetitive rounds. Each round is further divided into three periods

\section{- Reporting Period}

Static sensors report the events that have been detected but not yet processed in the period. Its length could depend on the frequency of event occurrence. This frequency can be learned by the statistics of event occurrence in previous rounds and used to adjust the length of the next reporting period.

\section{- Commanding Period}

The sink then computes how to assign MAM sensors to visit the events received in the reporting period, and transmits the assignment to the corresponding MAM sensors in the commanding period. Obviously, its length depends on the network diameter.

\section{- Dispatching Period}

In this period, MAM sensors move to their assigned event locations and perform analysis accordingly. The length of the dispatching period should include both the moving time of MAM sensors and the time spent to analyze events.

\subsubsection{Divided-and-Conquer ( $D \& C)$ Solution}

At first glance, one may intuitively adopt a divide-and conquer (D\&C) scheme by handling each attribute of event locations in a separate manner. Specifically, event locations are first clustered into groups based on their attributes. An instance of size $n$ can be divided into $b$ instances of size $n / b$, with " $a$ " of them needing to be solved. [a $\geq 1, b>1]$.

Wireless sensor networks (WSNs) offer a convenient way to monitor physical environments. WSNs are all considered static to continuously collect information from the environment. To improve the network capability in many aspects such as: automatic sensor node to deployments, flexible topology adjustment, and rapid event reaction. In this article to survey recent progress in mobile WSNs and compare works in this field in terms of their models and mobility management methodologies [1]. The discussion includes three aspects. Firstly, mobility management of mobile sensors for purposes of forming a better WSN, enhancing network coverage and connectivity, and relocating some sensors. Secondly, path-planning methods for data ferries to relay data between isolated sensors and to extend a WSN's lifetime. Finally, review some existing platforms of mobile WSNs.

To design and implementation of a home monitoring system based on hybrid sensor networks. The system follows a threelayer architecture which combines hybrid-node networking with web access [2]. An enhanced sensor node has been designed and fabricated to add controlled mobility to wireless sensor networks. The mobile node is capable of simple planar motions and is easy to be controlled through different user interfaces. A test bed including the static nodes as well as the mobile node has also been created for validating the basic functions of the proposed hybrid sensor network system. Network repair and event tracking capabilities of the mobile sensor node were tested. Stability in longtime home monitoring tasks was also verified.

Incorporating the environment-sensing capability of wireless sensor networks into video- based surveillance systems can provide advanced services at a lower cost than traditional surveillance systems [3]. The integrated mobile surveillance and wireless sensor system uses static and mobile wireless sensors to detect and then analyze unusual events in the environment.

An effective indoor localization method of hybrid RSSI/TDOA is proposed to reduce the big errors occurred during indoor RSSI localization and high cost paid by TDOA localization. It measures RSSI with iterative recursive weighted average filter, and polynomial model is obtained to fitting the RSSI measurement and to obtain polynomial model [4]. Furthermore the hybrid RSSI/TDOA localization algorithm is employed. The experiment results show that the proposed method of iterative recursive weighted average filter will improve the accuracy of RSSI localization on the condition of lower computation complexity, and the achieved localization is more accurate in the polynomial fitting than in the log-normal shadowing model. Moreover, the indoor location accuracy in the experiment is approximate 0.5 meter that satisfies the precision requirement of indoors location precision. 


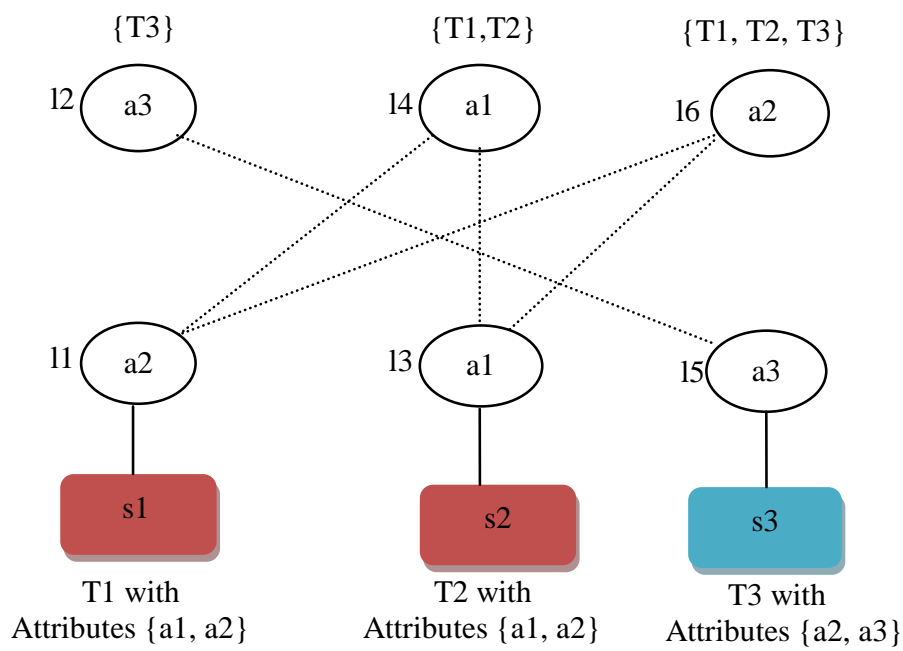

Fig 1: dispatching MAM sensors.

\section{CONCLUTION}

The conclusion intended to study of various platforms in wireless sensor network. In sensor dispatch problem in a WSN to solve this using the MAM sensor dispatch decision (MSDD) and the D\&C scheme could calculate a more balanced matching for the sensors. Heuristic is developed by reducing and balancing the energy consumption of MAM sensors. Dispatching sensors works to distribution of MAM sensors and event locations. In future research two phase heuristic methods may be used to dispatch problem. One heuristic for finding a Maximum Pareto-Optimal Matching uses a one-to-one manner to assign MAM sensors to visit event locations. At path efficiency of MAM sensors, so they are scheduled to reduce and balance the moving distances. Second, Constructing Multiple Spanning Trees a distributed version of our heuristic can be designed to let MAM sensors work in a decentralized fashion.

\section{REFERENCES}

[1] You-Chiun Wang, "A Two-Phase Dispatch Heuristic to Schedule the Movement of Multi-Attribute Mobile Sensors in a Hybrid Wireless Sensor Network," Mobile Computing, IEEE Transactions on, Vol. 13, no: 4, pp: 709- 722, 2014.

[2] Y.C. Wang, F.J. Wu, and Y.C. Tseng, "Mobility management algorithms and applications for mobile sensor networks," Wireless Comm. and Mobile Computing, vol. 12, no. 1, pp. 7-21, 2012.

[3] Y.C. Tseng, Y.C. Wang, K.Y. Cheng, and Y.Y. Hsieh, "iMouse: an integrated mobile surveillance and wireless sensor system," Computer, vol. 40, no. 6, pp. 60-66, 2007.

[4] N. Bartolini, T. Calamoneri, T.F. La Porta, and S. Silvestri, "Autonomous deployment of heterogeneous mobile sensors" IEEE Trans. Mobile Computing, vol. 10, no. 6, pp. 753-766, 2011
[5] D.P. Eickstedt, M.R. Benjamin, H. Schmidt, and J.J. Leonard, "Adaptive control of heterogeneous marine sensor platforms in an autonomous sensor network," Proc. IEEE/RSJ Int'l Conf. Intelligent Robots and Systems, pp. 5514-5521, 2006.

[6] M. Hofmeister, M. Kronfeld, and A. Zell, "Cooperative visual mapping in a heterogeneous team of mobile robots," Proc. IEEE Int'l Conf.Robotics and Automation, pp. 1491-1496, 2011.

[7] D.J. Abraham, K. Cechlarova, D.F. Manlove, and K. Mehlhorn, "Pareto optimality in house allocation problems," Proc. Int'l Conf.Algorithms and Computation, pp. 1163-1175, 2005.

[8] Z.J. Haas and B. Liang, "Ad hoc mobility management with uniform quorum systems," IEEE/ACM Trans. Networking, vol. 7, no. 2, pp.228-240, 1999.

[9] N. Li, J.C. Hou, and L.Sha, "Design and analysis of an MST-based topology control algorithm," IEEE Trans. Wireless Comm., vol. 4, no.3, pp. 1195-1206, 2005.

[10] Y.C. Wang, W.C. Peng, and Y.C. Tseng, "Energybalanced dispatch of mobile sensors in a hybrid wireless sensor network," IEEE Trans. Parallel and Distributed Systems, vol. 21, no. 12, pp. 1836-1850, 2010.

[11] H. Nishiyama , T. Ngo, N. Ansari, and N. Kato, "On minimizing the impact of mobility on topology control in mobile ad hoc networks, "IEEE Trans. Wireless Comm., vol. 11, no. 3, pp. 1158-1166, 2012

[12] L. Cheng, C.D. Wu, and Y.Z. Zhang, "Indoor robot localization based on wireless sensor networks," IEEE Trans. Consumer Electronics, vol.57, no. 3, pp. 10991104, 2011 